\title{
Performance Assessment of Darrieus Turbine with Modified Trailing Edge Airfoil for Low Wind Speeds
}

\author{
Palanisamy Mohan Kumar ${ }^{1,2 *}$, Rohan Kulkarni1, Narasimalu Srikanth', Teik-Cheng Lim² \\ ${ }^{1}$ Energy Research Institute, Nanyang Technological University, Singapore City, Singapore \\ ${ }^{2}$ School of Science and Technology, Singapore University of Social Sciences, Clementi, Singapore \\ Email: *pmkumar@ntu.edu.sg
}

How to cite this paper: Kumar, P.M., Kulkarni, R., Srikanth, N. and Lim, T.-C. (2017) Performance Assessment of Darrieus Turbine with Modified Trailing Edge Airfoil for Low Wind Speeds. Smart Grid and Renewable Energy, 8, 425-439.

https://doi.org/10.4236/sgre.2017.812028

Received: November 25, 2017

Accepted: December 25, 2017

Published: December 28, 2017

Copyright () 2017 by authors and Scientific Research Publishing Inc. This work is licensed under the Creative Commons Attribution International License (CC BY 4.0).

http://creativecommons.org/licenses/by/4.0/

\begin{abstract}
Darrieus wind turbines are simple lift based machines with exceptionally high efficiencies in terms of power coefficient compared to similar drag based vertical axis turbines. However, in low Reynolds numbers, a notable performance loss was reported. As a potential solution, truncated NACA 0018 airfoil (NACA 0018TC-39) has been introduced with baseline cavity modification to achieve better start-up characteristics and to enhance the low wind speed performance. The baseline cavity will provide an additional benefit of reverse drag at low TSR which is obligatory for low wind speed start-up. Numerical optimization has been carried out on the conceived airfoil NACA 0018TC-39 to find out the effective truncation percentage in terms of the chord. The numerical study has been extended to compare NACA 0018 and NACA 0018TC-39 airfoil for their aerodynamic performances in terms of lift, drag coefficients and separation characteristics. The NACA 0018TC-39 airfoil was incorporated within a non-swept straight bladed Darrieus turbine miniature to experimentally evaluate the performance in terms of dynamic power coefficient, dynamic torque coefficient and static torque coefficient and compared with conventional NACA 0018 airfoil at six different Reynolds numbers 178917, 193827, 208737, 223646, 238556 and 268376. The experimental contrast implied that NACA 0018TC-39 airfoil turbine yielded almost double power coefficients at low Reynolds number compared to conventional NACA 0018 airfoil without hampering its performance at higher Reynolds number.
\end{abstract}

\section{Keywords}

Darrieus Wind Turbine, Trailing Edge Cavity, Low Wind Speed and Truncated Airfoil 


\section{Introduction}

Wind energy is becoming the mainstream form of electrical energy generation with the total installed capacity of $486.8 \mathrm{GW}$ as of 2016 [1] and there has been an increasing trend. Small wind turbines are getting prime attention as the electricity generation mode shifts from big centralized power plants to isolated in-situ power generation applications such as telecommunications, remote sensing, and island powering. Along with other renewable sources, the distributed electricity generation minimizes the transmission and distribution losses [2]. Vertical Axis Wind Turbines (VAWTs) are increasingly popular for small power generation due to its unmatched qualities, such as simple structure [3], minimum footprint and Omni directionality. The blades can be either drag types in the case of Savonius turbines [4] or lift based as in the case of Darrieus turbines [5], later displaying a higher efficiency. Albeit, the drag based turbines are lower in efficiency [6], they have the better self-starting ability and operate seamlessly in highly turbulent and inconsistent wind, primary characteristics of an urban environment [7]. The Darrieus turbine with two straight blades is referred as H-Rotor and with multiple blades are referred as Giro mill. The vertical shaft of these devices makes it possible to position the generator at the base of the tower [8], which avoids additional loads on the structure and minimize mechanical losses. Darrieus turbines are visually attractive and silent, some vital aspects for urban installations [9] as they can extract more energy from the turbulent flows shed by buildings, compared to propeller types. With new airfoil design aimed at reducing the manufacturing cost of the blades, these turbines are affordable [10].

Apart from these advantages, the development of Darrieus turbines is hindered by their starting performance, pulsating torque, and poor performance in the low wind speed. Due to these haunting problems, the HAWTs are preferred for utility-scale power production. The efficiency of Darrieus turbines is comprised for the start-up in case of micro power generation. The problem of self-starting is further compounded by the inconsistent and low wind speed as these turbines are installed in locations of power requirement rather than optimum wind speed. Several solutions have been proposed to improve the low wind speed performance and a handful of attempts empowered the turbine with the self-starting capability and generated decent power at low winds, but at an increased cost. Rotor solidity is a critical factor influencing the rotor characteristics and the turbine performance in low wind speed. Numerous studies evaluate the performance of the Darrieus turbine with different number of blades and various chord lengths [11]. The starting characteristics are notably improved with an increase in number of blades, but the peak power coefficient is decreased. The computational studies revealed the wake interaction of preceding blades effectively reduces the AOA of the following blade resulting in decreasing of power coefficient [12]. A helical Darrieus turbine [13] can generate a decent starting torque for all azimuthal angles irrespective of the blade position. In additional blade, longevity can be obtained by the reduction of torque ripple. The 
starting torque generated by the helical turbines is not capable of pushing the rotor out of the dead band [14]. Hence the turbine either undergoes a slow acceleration or failure to start. Added, the manufacturing of helical blades is expensive and limits the application of inexpensive manufacturing process such as extrusion [15] for aluminum blades.

Blade thickness has a significant influence on both structural and aerodynamic properties. The past research predicts that $18 \%$ thick airfoil performs better for the Re range of $2 \times 10^{5}-3 \times 10^{5}$ with the maximum thickness of up to $28.5 \%$ [16] after which the power coefficient projects a decreasing trend. The effect of airfoil thickness on low wind operation [17] concludes that, though the power coefficient is lesser, the turbines will operate in a wider TSR. The blades with higher thickness are structurally sound withstanding higher centrifugal forces. Though the widely used airfoils are symmetric NACA profiles, cambered airfoil shows better starting characteristics and it seems logical to use symmetric airfoils as the angle of incidence is both positive and negative per cycle, the loss of power at the negative incidence in the downstream cycle is minimal. The study [14] experimentally compared NACA 0018 with varying camber Gottingen airfoils on the performance of Darrieus turbine concluding that peak power coefficient is obtained at lower TSR than symmetric airfoil. Cambered compared NACA63-015 was compared with NACA 0012, 0015 and 0018 and found that the cambered airfoil has smooth distribution of normal and tangential forces over wide range of azimuthal angles [18]. Cambered airfoils are commercially implemented along with high solidity to promote self-starting. Cambered airfoils are a better option compared to symmetric airfoils, yet a constant torque to accelerate the rotor at lower wind speed still lacks. In order to eliminate the negative torque on the downstream half cycle by the cambered blade and to get the best of the symmetric airfoil, morphing blades are proposed as a potential solution to dynamically vary the camber [19]. The morphing blades are successfully implemented in large horizontal axis wind turbine to alleviate the load at high wind loads [20]. Airfoil with trapped vortex concept was put forward for the improvement of starting torque [21] and verified computationally with conventional NACA based rotor [22] early studies on the morphing blades are with flexible sails and sheet metal plates, which have not yielded fruitful results to conduct further research. The modern elastomeric material such as Tecoflex 80 A [23] together with low cost electronics and actuation systems makes it applicable for higher capacity turbines. To reduce the complexity and the requirement of special elastomeric materials, the trailing edge flaps are considered to be an alternative solution. The technology was well developed and initially applied on helicopter rotors to reduce noise, vibrations and in the race car applications for creating a high nose down pitching moment. Early study with hinged flap on Darrieus turbine [24] with NACA0012 indicated a 10\% increase in power coefficient at higher TSR compared to the conventional blade. The power improvement is attributed to the delay in the dynamic stall and reduction in the down- 
stream drag with favorable AoA achieved with the flaps.

\section{Objective of the Present Work}

The literature summarizes the feasible solutions to improve the power performance of the Darrieus turbines. Most of the solutions proposed, either demands the addition of new components or highly complex mechanism contributing the increase in weight or economically not viable. Hence the sole objective of the current study is in search of a simple solution that can be incorporated economically to exploit these turbines at low wind speeds. Since the drag type blades generate higher torque at low wind speed, any form of drag in the direction of rotation could be an added advantage. The study emphasis on modifying the existing airfoil to create a reverse drag at low wind speed without compromising the lift and torque at rated wind speed.

\section{Airfoil Design}

The truncated airfoil finds its application in wind turbines especially in the large HAWTs at the blade root region. The transition region from airfoil to cylindrical root requires thicker airfoil up to $40 \%$ to withstand the high bending moment as the structural properties crucial than the aerodynamic properties, where the truncated trailing edge airfoil (TTE) are indispensable.

The structurally superior blunt airfoil has some excellent aerodynamic features which makes it superior to conventional airfoil. A research from NASA along with Ohio State University suggested that blunting the $40 \%$ thick airfoil increases the maximum lift coefficient by almost $100 \%$. Purpose of the truncation process is to eliminate the sharp and steep curvature at the trailing edge portion which will in turn reduces the adverse pressure gradient caused in that area due to full pressure recovery. The reduced adverse pressure gradient helps in delaying the flow separation which is a prime concern in low-speed flows [25].

The airfoil design is the most important aspect of this research. Typically, blunt trailing edge airfoils are created either by truncation or flatback. Truncating the airfoil involves cutting off some portion of the airfoil from trailing edge and making it blunt that may modify the camber. An alternate solution is flatback airfoils, which essentially involves adding sufficient thickness on both pressure and suction side symmetrically preserving its camber. Since this study involves the symmetrical airfoil, the methodology used is simple airfoil truncation. Hoerner [26] showed few experimental findings for truncated trailing edge airfoils, including the results for the symmetric Go"-490 airfoil. At Reynolds number of 500,000 the lift coefficient of this symmetric airfoil seemed to increase with the truncation percentage up to a limit. The maximum lift coefficient increased by almost $45 \%$ when the airfoil was truncated at $35 \%$. According to Tangler and Somers [27], sharp trailing-edge airfoils with $t / c<0.25$ can be modified to have lifting properties that are vastly susceptible to surface disturbances. 
Hence a simple yet effective guideline for blunt trailing edge airfoils is to restrict the difference between the maximum thicknesses to trailing edge thickness to $\sim 20 \%$ of chord length. In doing so, the adverse pressure gradient on later portion of the suction surface is controlled, which in turn recovers the expected lifting properties under disturbed conditions. Based on this rule of thumb the airfoil designing has been done in this research. Baker [26] described, the flatback (FB) series of airfoils was discovered by mixing the suction side shape drawn from the thick, high lift inboard NREL airfoils, and a structurally superior pressure side derived from the LS-1 series airfoils. The family of FB airfoils was developed by Sandia National Laboratory in collaboration with the University of California-Davis as part of the Blade System Design Study (BSDS). The strategy behind the new airfoil design is to dramatically increase the output torque at low wind speed and low Tip Speed Ratio (TSR). The non-cumbersome way is to intelligently use the airfoil drag if generated in the direction of rotation of the rotor. To make it possible, a drag creating feature should be introduced at the trailing edge of the airfoil, as the flow is towards leading edge from trailing edge when the blade retreats from the incoming wind. A thick trailing edge will create more base drag compared to sharp trailing edge due to vortex shedding. Gangad haracharya [28] suggests that, the TTE airfoil generates higher lift at high Angle of Attack (AOA), where the conventional airfoil could have stalled. A major drawback of TTE airfoil is the abrupt stall which may induce instability and vibrations if incorporated in Darrieus turbine. For smooth stall and to reduce the base drag, Baker [29] introduced splitter plates mounted perpendicular to the trailing edge. $25 \%$ reduction in drag was reported for single splitter plate. The study extends with double splitter plate similar to the cavity and the results indicated a smooth stall. For the current study, the blunt trailing edge is created from conventional NACA 0018 airfoil and a cavity is excavated inside blunt trailing edge. To incorporate a cavity with sufficiently large radius to maximize the Darrieus turbine performance, the trailing edge should be relatively thick and this necessitates the airfoil to be truncated. The truncation \% from the trailing edge is a subject matter of subjected of optimization. The airfoils under investigation are shown in Figure 1.

\section{Computational Study}

The study is performed in a two-dimensional domain. The computational domain consists of a $\mathrm{C}$ type grid. The distances from the airfoil leading edge to the domain inlet and outlet are $10 \mathrm{c}$ and $25 \mathrm{c}$, with a domain width of $20 \mathrm{c}$, whereas $\mathrm{c}$ is the airfoil, (blockage ratio 5\%). The computational grid consists of quadrilateral cells as illustrated in Figure 2, where a boundary layer grid and restraining the $y+$ value below 1 on the walls. The two-dimensional grid has approximately 80,000 cells. The grid resolution resulted from a grid-convergence study using three grids generated based on an overall linear factor $\sqrt{ } 2$ for coarsening and refining. The boundary conditions are set as constant inlet velocity of $44 \mathrm{~m} / \mathrm{s}$, 

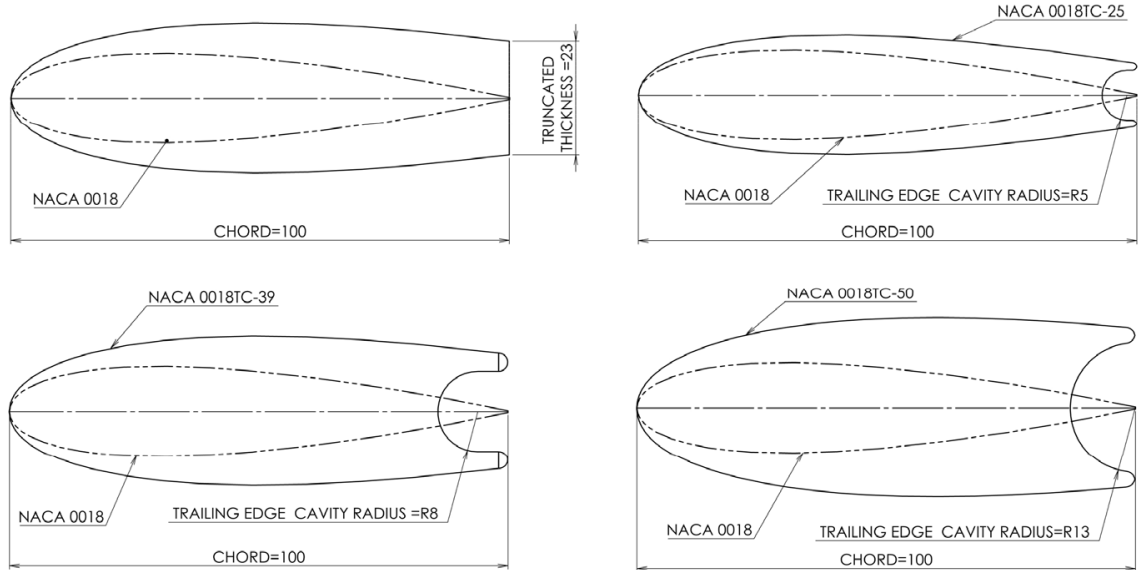

Figure 1. Different truncated airfoil comparisons with the conventional NACA 0018 airfoil.
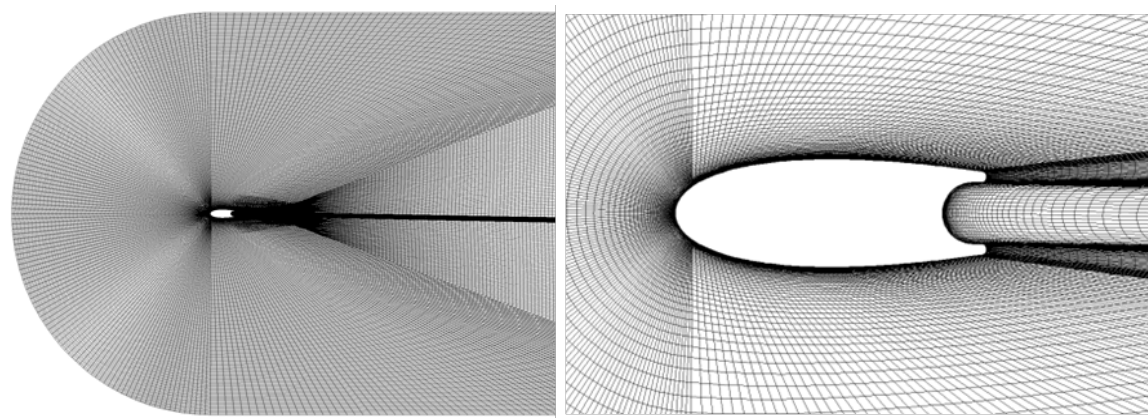

Figure 2. NACA 0018TC-39 static airfoil analysis mesh in ICEM CFD.

zero static gauge pressure at the outlet, symmetry on the sides and a no-slip condition on the airfoil. The approach-flow uniform total turbulence intensity at the domain inlet is 5\%. Reynolds Averaged Navier-Strokes (RANS) calculations are performed using the commercial computational software package ANSYS/Fluent 16. Both spatial and temporal discretization is second-order and pressure-velocity coupling is done with coupled scheme. Turbulence is modelled using the 2-equation $\mathrm{k}-\omega$ SST model. As we can observe from the vector plot Figures 3(a)-(d) that at lower angle of attack, the flow is getting curled inside the baseline cavity essentially creating a pair of counter-rotating vortices trapped inside the cavity. Due to trapping of these shaded vortices, the vortex street formed behind the thick trailing edge is restricted in one manner. This phenomenon assists in reducing the wake region width behind the airfoil up to certain extent. As the AOA increases, the rotating vortex gradually disappears, and the flow separation moves towards the leading edge resulting in higher drag.

\section{Lift and Drag Characteristics}

From the obtained curves, it is evident that the lifting performance of $25 \%$ truncated airfoil is superior to rest but will result in smaller baseline cavity. The 50\% truncated airfoil should produce maximum lift according to truncated airfoil 


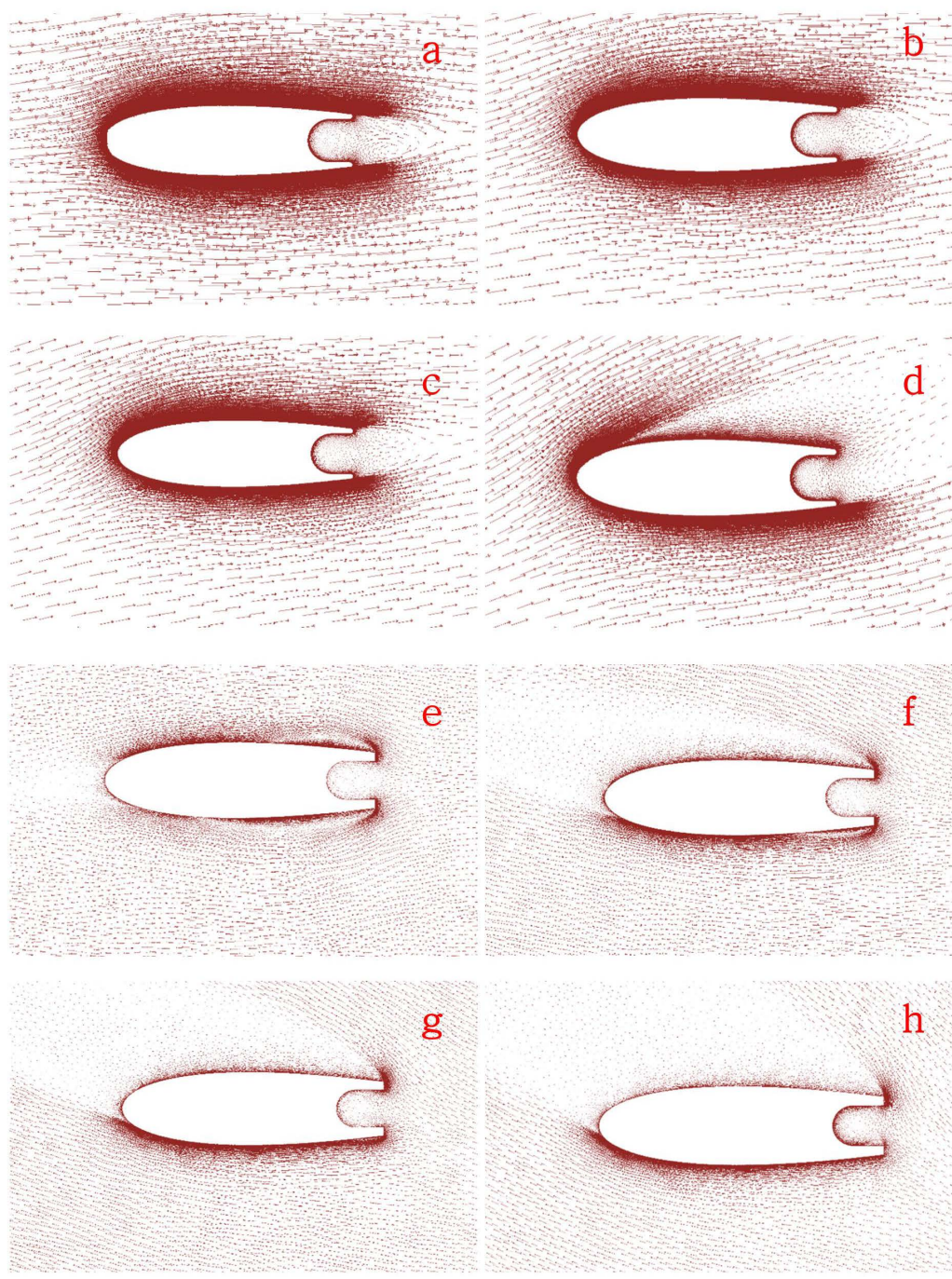

Figure 3. Vector representation of NACA0018TC-39 airfoil in forward flow (3(a)-(d)) and reverse flow (3(e)-(h)) (AoA $9^{\circ}$ (a) (e), $10^{\circ}$ (b) (f), $20^{\circ}$ (c) (g), 30 (d) (h)).

theory but may lead to early separation at high AOA. The separation can be attributed to high leading-edge thickness which presents itself as bluff body. All these factors considered, the overall aerodynamic performance of the $39 \%$ truncated airfoil is much more effective than rest of the configurations for most of the angle of attack range. Also, larger baseline cavity will allow the airfoil to produce larger reverse drag in low TSR.

To quantify the reverse drag on the airfoil, the computational study has been done on the optimized airfoil. Similar grid size has been adapted for this study with $y+$ value less than 1 . The results are shown in the vector contours Figure 3(e)-(h). For the Reynolds number 300,000 the drag coefficient for the airfoil in a reverse manner found out to be 0.08 which is exceptionally high. As the angle of attack increases to 10,20 and 30 , the drag coefficient continues to increase, $0.17,0.22$, and 0.26 as shown in Figure 4. These high drag coefficients in backward 


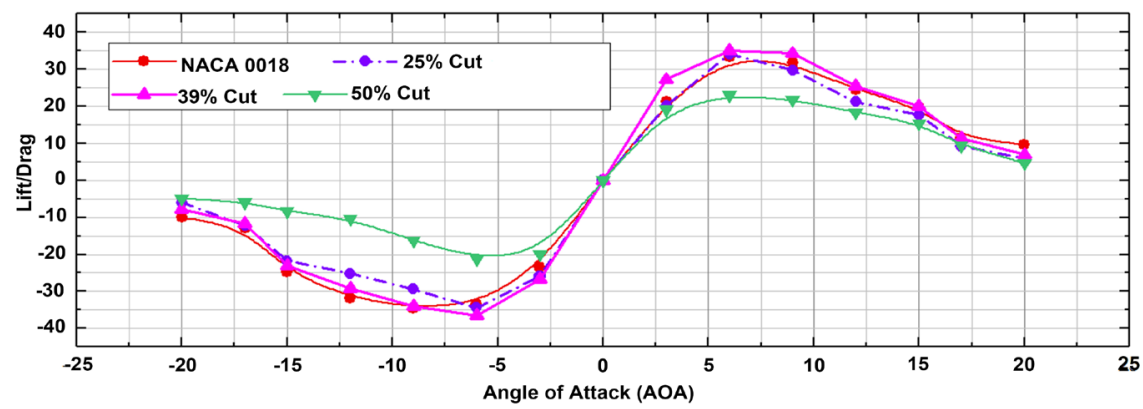

Figure 4. Comparision of lift and drag characteristics of truncated airfoils.

direction provide high moments which add up to the torque produced by lifting blades together yielding better starting properties.

\section{Experimental Setup and Procedure}

The blades of both the rotors were made by $3 \mathrm{D}$ printing and polished for the smooth surface finish, as Re is highly sensitive to surface roughness. An end plate on top and bottom supports the blades of both the rotors in order to eliminate the struts and loss of torque due to the strut drag. The endplate prevents the blade tip vortices, which is a substantial loss for the turbine of low aspect ratio. The top and bottom end plates are secured by shaft collar to $20 \mathrm{~mm}$ aluminum shaft. The rotor shaft was supported on the bottom end with deep groove ball bearing and top end with aspherical ball bearing to accommodate any shaft misalignment. The bearings are periodically lubricated to minimize the friction. The fabricated NACA 0018TC-39 bladed turbine was tested in the (Energy Research Institute At Nanyang) ERIAN subsonic wind tunnel with the test section of $0.7 \mathrm{~m} \times 0.7 \mathrm{~m}$. The wind tunnel was equipped with axial flow fan (Multi-Wing) of $0.9 \mathrm{~m}$ diameter powered by a permanent magnet motor of $5 \mathrm{~kW}$ capacity. The wind tunnel can generate maximum free stream wind speed of 15 $\mathrm{m} / \mathrm{s}$ with the turbulent intensity of less than $\pm 1 \%$. The wind speed is measured by the hot wire anemometer at a distance of $900 \mathrm{~mm}$ from the rotor center to avert any flow disturbance upstream of the turbine. The braking torque was applied by magnetic particle brake (Placid Industries) to vary the TSR. The generated torque was measured through Lorenz torque sensor and the rotational speed was measured by the proximity sensor. The digital pulses from the proximity sensor are counted per minute by data acquisition system to output as rotor speed. All the instruments and sensor are shown in Figure 5. The rotor dimensions of both the configurations are shown in Table 1 . The wind tunnel facility is shown with the mounted rotors are displayed in Figure 6.

\section{Results and Discussion}

\subsection{Data Reduction}

The performances of proposed Darrieus turbine with NACA0018TC-39 and conventional Darrieus with NACA0018 airfoil are compared in terms following coefficients as given. 


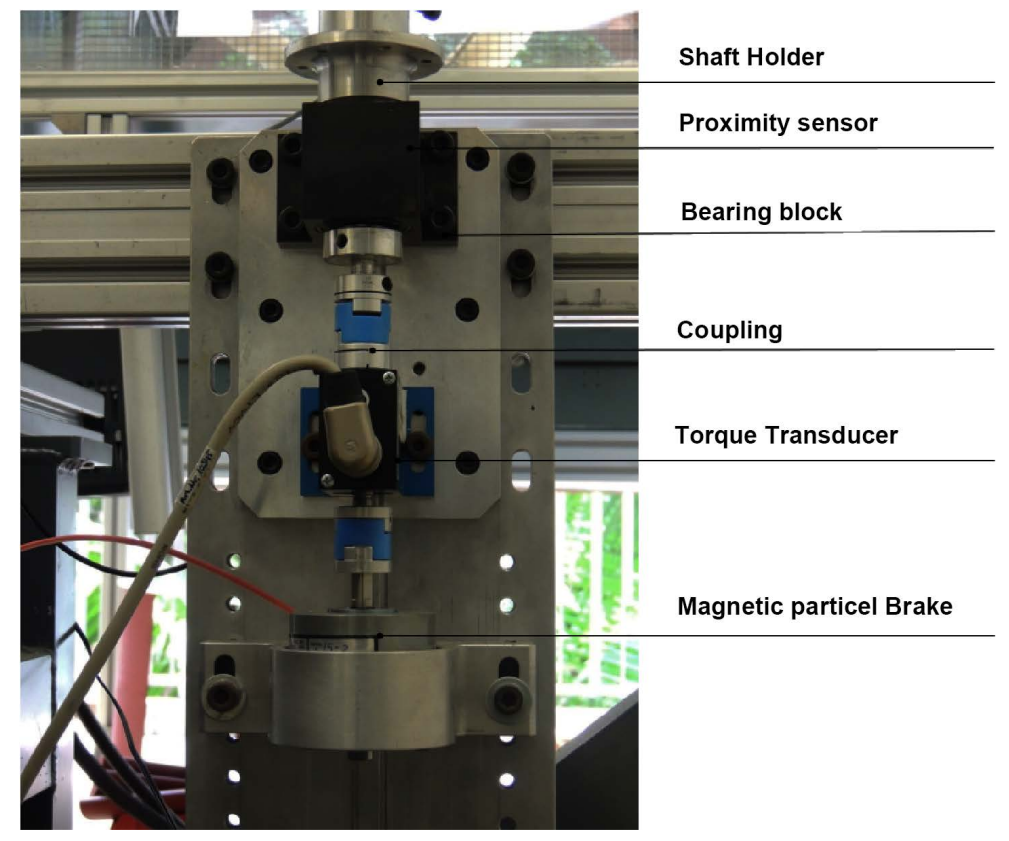

Figure 5. Instrumentation of the Test Setup.

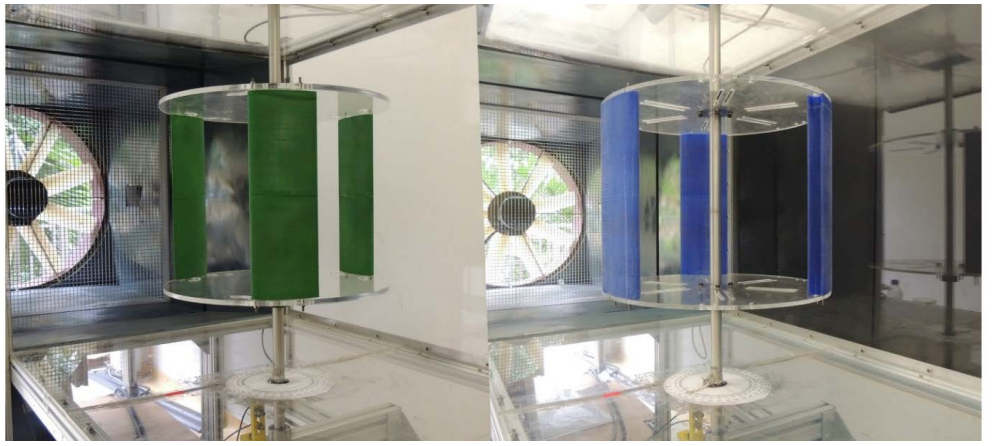

Figure 6. H-turbine prototype mounted in wind tunnel with conventional NACA 0018 blades (left) and NACA 0018TC-39 blades (right).

Table 1. Test rotor details.

\begin{tabular}{cccc}
\hline Design parameters & NACA 0018 & NACA 0018TC-39 & Units \\
\hline Rotor Diameter & 400 & 400 & $\mathrm{~mm}$ \\
Rotor Height & 300 & 300 & $\mathrm{~mm}$ \\
Solidity & 1.5 & 1.5 & $\mathrm{~mm}$ \\
Center Shaft Diameter & 20 & 20 & - \\
Operating ReNumber & $100,000-280,000$ & $100000-280,000$ & $\mathrm{~mm}$ \\
Airfoil Chord & 100 & 100 & - \\
Number of Blades & NACA 0018 & NACA $0018 \mathrm{TC}-39$ & $\mathrm{~mm}$ \\
End plate diameter & 3 & 3 & $\mathrm{~mm}$ \\
End plate thickness & 420 & 420 & - \\
Support & 8 & 8 & \\
\hline
\end{tabular}




$$
C_{t}=\frac{T}{0.5 * R * \rho * v^{2} * A_{S}}
$$

$C_{t}$ is the dynamic torque coefficient, $T$ is the dynamic torque on the turbine shaft $(\mathrm{N}-\mathrm{m}), \quad \rho$ is the density of air $\left(\mathrm{kg} /\left(\mathrm{m}^{\wedge} 3\right)\right), A_{S}$ is the planform area of the blade $\left(\mathrm{m}^{2}\right), \mathrm{R}$ is the radius of the turbine $(\mathrm{m})$.

$$
C_{p}=\frac{T * \omega}{0.5 * R * \rho * v^{3} * A_{S}}
$$

where, $C_{p}$ is the power coefficient, $\omega$ is the angular velocity ( $\left.\mathrm{rad} / \mathrm{s}\right)$

$$
C_{T S}=\frac{T_{s}}{0.5 * R * \rho * v^{2} * A_{S}}
$$

$C_{T S}$ denotes static torque co-efficient and $T_{S}$ is static torque $(\mathrm{N}-\mathrm{m})$. The blockage correction has not been considered for the current study as the objective is to compare the two configurations rather than assessing the definite performance of a specific configuration.

\subsection{Power Coefficient}

Dynamical wind turbine performances are obtained by computing the instantaneous torque coefficient $C_{t}$. Figure 7 shows the dynamic power coefficient for both NACA0018TC-39 airfoil and NACA 0018 airfoil in comparison. For a wide range of Re, the high-power coefficient is achieved by NACA0018TC-39 at lower TSR values signifying prominent increment in starting torque. For Re 178,917, for conventional turbine, highest $\mathrm{Cp}$ of 0.09 is achieved at TSR of 1.1 while for the same Re, for the NACA 0018TC-39 bladed configuration, the highest Cp of 0.13 was achieved at TSR of 1.07. For the lower values of TSR, NACA 0018TC-39 bladed rotor continues to perform significantly better than conventional configuration in terms of power coefficient. For Re 268,376 where the performance of NACA 0018TC-39 bladed turbine is still superior but the increment is not very high. The possible reason could be the wake produced by the oscillating vortex street behind blunt trailing edge yields fluctuating lifting characteristics causing the turbine to deviate from the design point. However, the thicker airfoil continues to provide the high lifting coefficients making up for loss created due to vortex shedding. At lower TSR values the NACA 0018TC-39 bladed rotor provides exceptionally good characteristics due to additional reverse drag component obtained due to the baseline cavity which adds up to the lift force eventually providing additional useful starting torque. The overall $\mathrm{Cp}$ value for both the configurations is reduced to some extent due to significant frictional torque in the measurement trains such as bearing and couplings.

\subsection{Static Torque Coefficient}

Darrieus turbines are notorious for not self-starting as the blades undergo static stall due to high AOA. It is nearly impossible to self-start the turbine without any concession on power coefficient. The iota lift generated by the blades at rest 

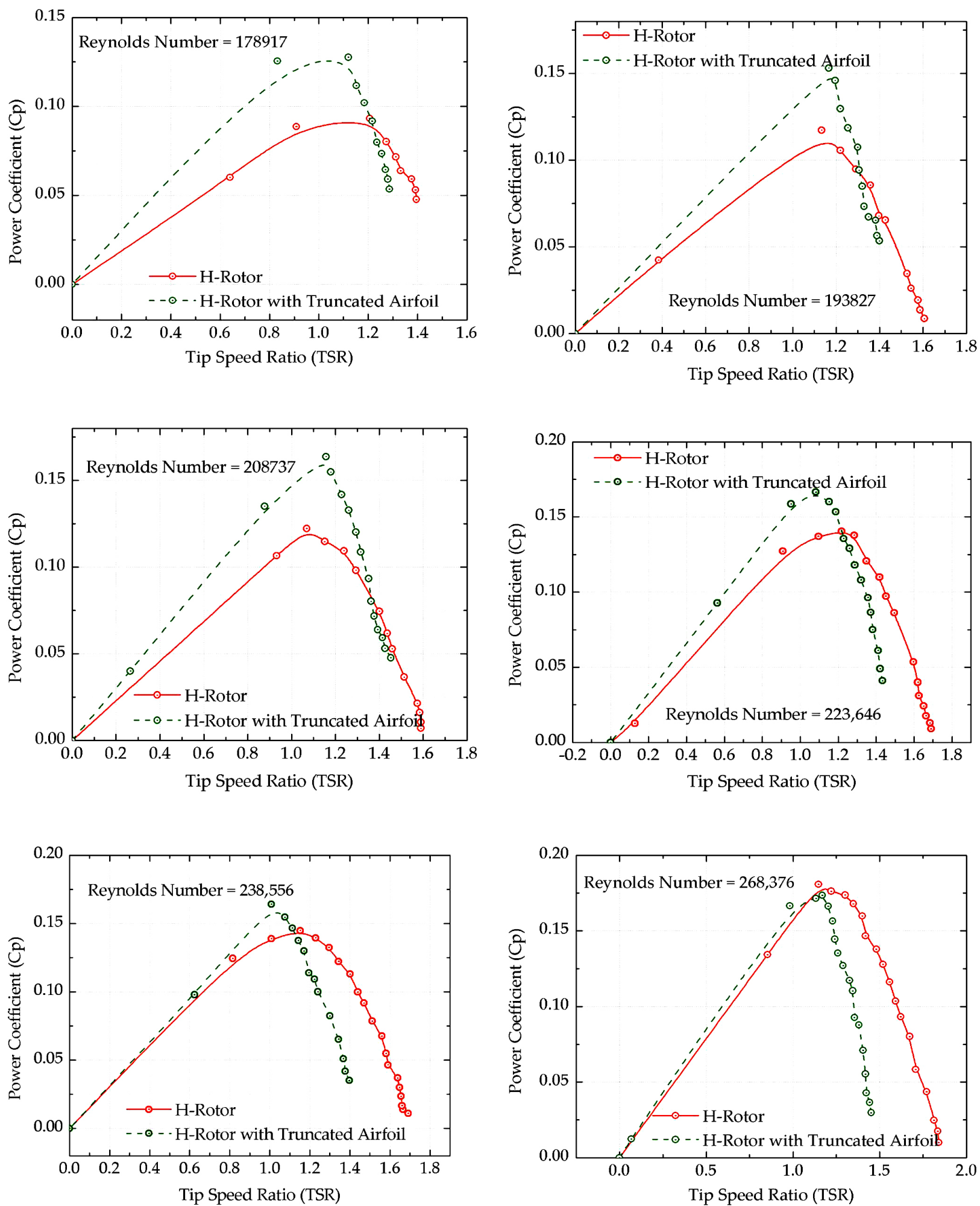

Figure 7. Power coefficient curve at different Reynolds numbers.

is inadequate to overcome the self-inertia of the turbine as well as instrumental frictional losses. Hence it is imperative to study the behavior of blades relative to the incoming wind when the wind turbine is standstill and ensure that the blades 
generate ample torque to accelerate the rotor beyond the dead band and drivetrain resistance.

The starting issues are compounded by the random position of the rotor between 0 and $2 \pi$. Hence to establish an apparent correlation between starting torque and the free stream wind, it is recommended to measure the torque generated for all the azimuthal angles. Static torque coefficient is a vital parameter to judge the starting characteristics of the Darrieus wind turbine which is characterized by the net torque generated by the blades at rest.

At very low TSR the output a negligible torque and the cavity introduction is anticipated to boost the torque to significant value. Both the configurations are subjected to static torque coefficient measurement for $360^{\circ}$ with $15^{\circ}$ increments computed as per the Equation (3). The results reinforce the hypothesis that the trailing edge cavity will promote self-starting and increase the low rpm at low wind speed. For the tested Re, the NACA 0018TC-39 rotor demonstrates a higher torque for most of the azimuthal position than NACA0018 rotor.

Another notable behavior exhibited by NACA0018TC-39 rotor is that static torque is distributed from $0^{\circ}$ to $360^{\circ}$, rather than three peaks at an interval of $120^{\circ}$ by conventional Darrieus. The peak torque will lead to static stall if AOA is large at the renewed position of blades A smooth positive torque distribution aids the rotor to accelerate to the rated rpm as illustrated in Figure 8.

Comparing the torque coefficient values, the NACA 0018TC-39 bladed turbine accomplishes $21 \%$ at $178,917,23 \%$ at 193827 and $5 \%$ at 266,896 more static torque than theNACA0018 rotor. In the case of NACA 0018TC-39 for the Re 208737, the minimum static torque coefficient of 0.02 accelerates the rotor beyond stall, for the investigated Re, the NACA 0018TC-39 has better starting and low wind speed performance than the conventional configuration.

\section{Conclusion}

The present work is focused on the research problem of new airfoil design, analysis and its comparison with conventional airfoil for enhancing the weak wind flow performance of straight bladed Darrieus Turbine in range of $\operatorname{Re}\left(1 \times 10^{5}-3\right.$ $\times 10^{5}$ ) which is typically experienced by various small VAWTs. The old school NACA 0018 airfoil was modified and optimized as NACA 0018TC-39 for its structural and aerodynamic benefits and subjected to extensive wind tunnel tests. Computational simulations are employed to optimize the truncation location on the chord and their influence on lift and drag coefficients. Proposed turbine displayed excellent performance for the tested low Re with enhanced lift characteristics and a significant rise in torque coefficient. The power coefficient of NACA0018TC-39 is $12.6 \%$ at $178,917,1.6 \%$ at 238,566 higher than conventional NACA 0018 airfoil. The difference in power coefficient between the investigated airfoil progressively diminishes as the Re increases. This evidences that the truncated airfoil manifests itself as a conventional airfoil in higher Re with only a mild influence on the lift and drag characteristics. To conclude, the new 


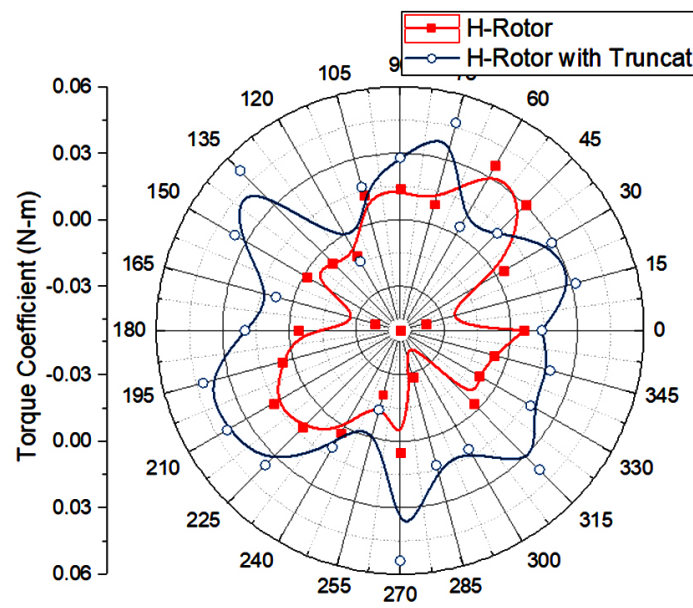

(a)

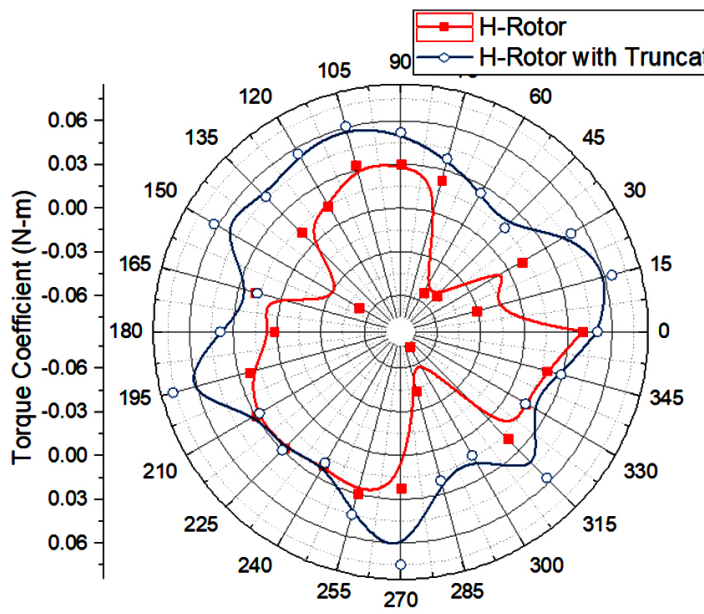

(c)

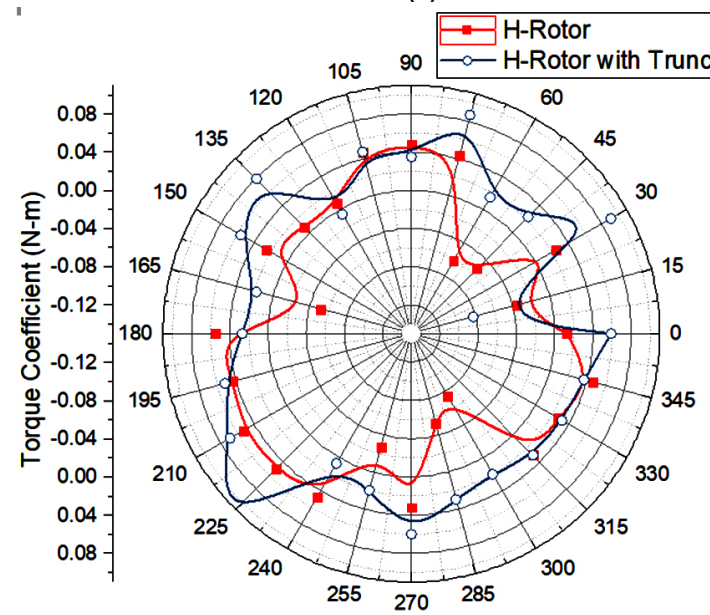

(e)

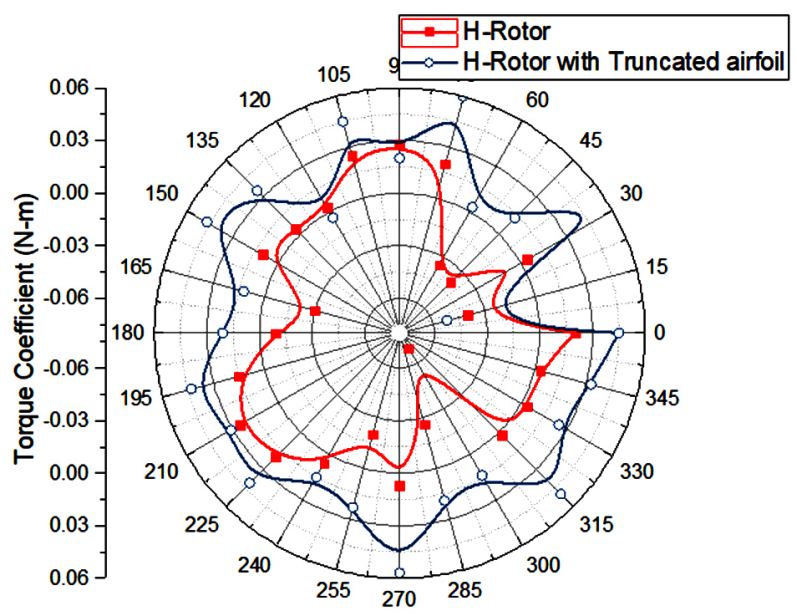

(b)

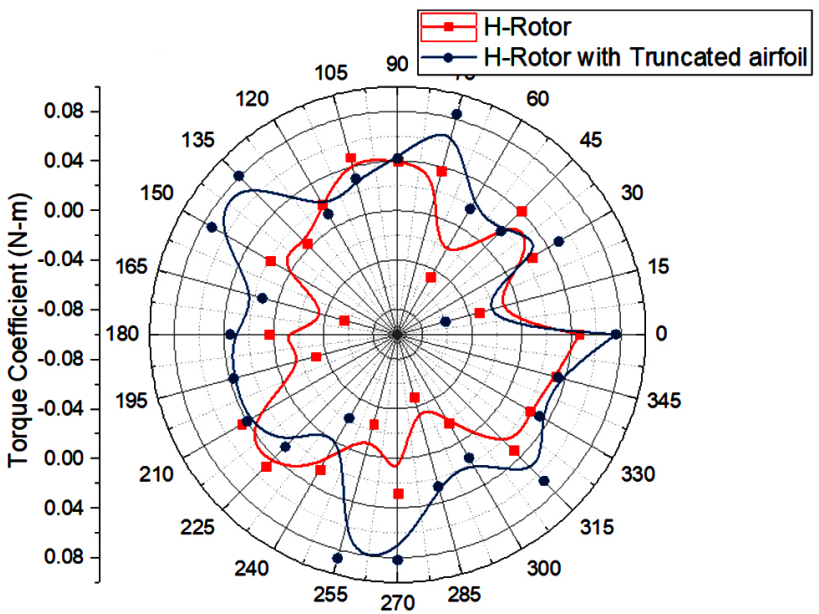

(d)

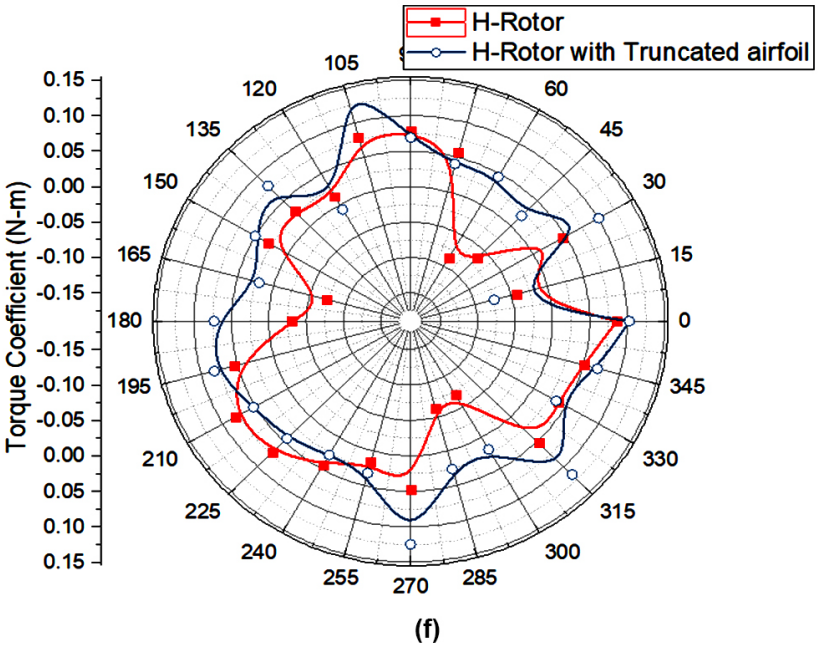

Figure 8. Static torque coefficient variation with the azimuthal angle for different Reynolds numbers. (a) $\operatorname{Re}=178,917$; (b) $\operatorname{Re}=$ 193,827 ; (c) $\operatorname{Re}=208,737 ;$ (d) $\operatorname{Re}=223,646$; (e) $\operatorname{Re}=238,556$; (f) $\operatorname{Re}=268,376$.

Darrieus rotor crafted with NACA 0018TC-39 can operate in whole wind spectrum efficiently from the power generation perspective. The enhanced starting characteristics ensure the delivery of reasonable wattage even at wind speed of 2 
$\mathrm{m} / \mathrm{s}$ or less compared to the cut in wind speed of $3.5 \sim 4.5 \mathrm{~m} / \mathrm{s}$ for the conventional Darrieus turbine. The truncated airfoil with cavity can be further optimized and tested for higher Re for the application in large VAWTs.

\section{Acknowledgements}

This research is supported by the National Research Foundation, Prime Minister's Office, Singapore under its Energy Innovation Research Programme (EIRP Award No. NRF2013EWT-EIRP003-032: Efficient Low Flow Wind Turbine).

\section{References}

[1] Redlinger, R., Andersen, P. and Morthorst, P. (2016) Wind Energy in the 21st Century: Economics, Policy, Technology and the Changing Electricity Industry. Springer, Berlin.

[2] Ward, J.B., Eaton, J.R. and Hale, H.W. (1950) Losses in Power Transmission Networks. Electrical Engineering, 69, 451-451. https://doi.org/10.1109/EE.1950.6433860

[3] Tjiu, W., Marnoto, T., Mat, S., Ruslan, M.H. and Sopian, K. (2015) Darrieus Vertical Axis Wind Turbine for Power Generation II: Challenges in HAWT and the Opportunity of Multi-Megawatt Darrieus VAWT Development. Renewable Energy, 75, 560-571. https://doi.org/10.1016/j.renene.2014.10.039

[4] Wenehenubun, F., Saputra, A. and Sutanto, H. (2015) An Experimental Study on the Performance of Savonius Wind Turbines Related with the Number of Blades. Energy Procedia, 68, 297-304. https://doi.org/10.1016/j.egypro.2015.03.259

[5] van Bussel, G.J.W. (2013) Electricity Generation Electricity Generation with Small Wind Turbines Wind Turbine, Renewable Energy Systems. Springer, Berlin, 696-713.

[6] Abraham, J.P., Plourde, B.D., Mowry, G.S., Minkowycz, W.J. and Sparrow, E.M. (2012) Summary of Savonius Wind Turbine Development and Future Applications for Small-Scale Power Generation. Journal of Renewable and Sustainable Energy, 4, Article ID: 042703. https://doi.org/10.1063/1.4747822

[7] Karthikeya, B.R., Negi, P.S. and Srikanth, N. (2016) Wind Resource Assessment for Urban Renewable Energy Application in Singapore. Renewable Energy, 87, 403-414. https://doi.org/10.1016/j.renene.2015.10.010

[8] Eriksson, S., Bernhoff, H. and Leijon, M. (2008) Evaluation of Different Turbine Concepts for Wind Power. Renewable and Sustainable Energy Reviews 12, 1419-1434. https://doi.org/10.1016/j.rser.2006.05.017

[9] Bahaj, A.S., Myers, L. and James, P.A.B. (2007) Urban Energy Generation: Influence of Micro-Wind Turbine Output on Electricity Consumption in Buildings. Energy and Buildings, 39, 154-165. https://doi.org/10.1016/j.enbuild.2006.06.001

[10] Kumar, M., Surya, M.M.R., Sin, N.P. and Srikanth, N. (2017) Design and Experimental Investigation of Airfoil for Extruded Blades. International Journal of Advances in Agricultural and Environmental Engineering (IJAAEE), 3, 2349-1523.

[11] Worasinchai, S., Ingram, G.L. and Dominy, R.G. (2014) The Physics of H-Darrieus Turbine Starting Behaviour. Proceedings of the ASME Turbo Expo, Dusseldorf, 16-20 June 2014. https://doi.org/10.1115/GT2014-25461

[12] Scheurich, F., Fletcher, T.M. and Brown, R.E. (2011) Simulating the Aerodynamic Performance and Wake Dynamics of a Vertical-Axis Wind Turbine. Wind Energy, 
14, 159-177. https://doi.org/10.1002/we.409

[13] Gorlov, A. (1998) Development of the Helical Reaction Hydraulic Turbine. Final Technical Report, 1 July 1996-30 June 1998, Northeastern University, Boston. https://doi.org/10.2172/666280

[14] Baker, J.R. (1983) Features to Aid or Enable Self Starting of Fixed Pitch Low Solidity Vertical Axis Wind Turbines. Journal of Wind Engineering and Industrial Aerodynamics, 15, 369-380. https://doi.org/10.1016/0167-6105(83)90206-4

[15] Blackwell, B.F. and Reis, G.E. (1974) Blade Shape for a Troposkien Type of Vertical-Axis Wind Turbine, Sandia Labs, Albuquerque.

[16] Healy, J.V. (1978) The Influence of Blade Thickness on the Output of Vertical Axis Wind Turbines. Wind Engineering, 2, 1-9.

[17] Parchen, R., Bruggeman, J.C. and Dassen, A.G.M. (1996) The Effect of the Blade Thickness of Wind Turbine Blades on the Noise Due to Inflow Turbulence. Acustica, 82, S82-S82.

[18] Zervos, A. and Mudry, M. (1988) Aerodynamic Design and Testing of Blade Profiles for Vertical Axis Wind Turbines. Commission of the European Communities, Contractors' Meeting, 309-320.

[19] Lachenal, X., Daynes, S. and Weaver, P.M. (2013) Review of Morphing Concepts and Materials for Wind Turbine Blade Applications. Wind Energy, 16, 283-307. https://doi.org/10.1002/we.531

[20] Zayas, J.R., Van Dam, C.P., Chow, R., Baker, J.P. and Mayda, E.A. (2007) Active Aerodynamic Load Control for Wind Turbine Blades. Load Control for Wind Turbine Blades, European Wind Energy Conference.

[21] Kumar, M., Mohan Ram Surya, M. and Srikanth, N. (2017) On the Improvement of Starting Torque of Darrieus Wind Turbine with Trapped Vortex Airfoil. IEEE International Conference on Smart Grid and Smart Cities, Singapore, 23-26 July 2017, 120-125.

[22] Kumar, M., Mohan Ram Surya, M. and Srikanth, N. (2017) Comparative CFD Analysis of Darrieus Wind Turbine with NTU-20-V and NACA0018 Airfoils. IEEE International Conference on Smart Grid and Smart Cities, Singapore, 23-26 July 2017, 108-114.

[23] Maheri, A. and Isikveren, A.T. (2010) Performance Prediction of Wind Turbines Utilizing Passive Smart Blades: Approaches and Evaluation. Wind Energy, 13, 255-265. https://doi.org/10.1002/we.340

[24] Yang, Y., Li, C., Zhang, W., Guo, X. and Yuan, Q. (2017) Investigation on Aerodynamics and Active Flow Control of a Vertical Axis Wind Turbine with Flapped Airfoil. Journal of Mechanical Science and Technology, 31, 1645-1655. https://doi.org/10.1007/s12206-017-0312-0

[25] Law, S.P. and Gregorek, G.M. (1987) Wind Tunnel Evaluation of a Truncated NACA 64-621 Airfoil for Wind Turbine Applications.

[26] Baker, J.P., Mayda, E.A. and Van Dam, C.P. (2006) Experimental Analysis of Thick Blunt Trailing-Edge Wind Turbine Airfoils. Journal of Solar Energy Engineering, 128, 422-431. https://doi.org/10.1115/1.2346701

[27] Tangler, J.L. and Somers, D.M. (1995) NREL Airfoil Families for HAWTs. National Renewable Energy Lab., Golden.

[28] Gangadharacharya, K.B. (2015) Vortex Shedding and Aerodynamic Drag on Truncated Trailing Edge Airfoil.

[29] Baker, J.P. (2009) Drag Reduction of a Blunt Trailing-Edge Airfoil. University of California, Davis. 